\title{
Polypharmacy and injurious falls in older adults: a nationwide nested case-control study
}

This article was published in the following Dove Press journal:

Clinical Epidemiology

\author{
Lucas Morin' \\ Amaia Calderon Larrañaga' \\ Anna-Karin Welmer ${ }^{1-4}$ \\ Debora Rizzuto' \\ Jonas W Wastesson' \\ Kristina Johnell ${ }^{5}$ \\ 'Aging Research Center, Karolinska \\ Institutet and Stockholm University, \\ Stockholm, Sweden; ${ }^{2}$ Division of \\ Physiotherapy, Department of \\ Neurobiology, Care Sciences and Society, \\ Karolinska Institutet, Stockholm, Sweden; \\ ${ }^{3}$ Allied Health Professionals, Function \\ Area Occupational Therapy \& \\ Physiotherapy, Karolinska University \\ Hospital, Stockholm, Sweden; \\ ${ }^{4}$ Stockholm Gerontology Research \\ Center, Stockholm, Sweden; \\ ${ }^{5}$ Department of Medical Epidemiology \\ and Biostatistics, Karolinska Institutet, \\ Stockholm, Sweden
}

Objective: To determine whether or not the exposure to multiple drugs (polypharmacy) increases the risk of fall-related injury among older adults, beyond the effect of fall-risk increasing drugs and chronic multimorbidity.

Methods: Nested case-control study using linked register data with national coverage in Sweden. We defined cases as older adults ( $\geq 70$ years) who had an incident non-elective admission due to a fall between 1 January and 31 December 2013. Cases were matched 1:1 on sex, age and index date to randomly selected controls from the general population. The number of prescription drugs during the 7 days preceding the index date was the main exposure.

Results: A total of 49,609 cases were included and matched to an equal number of controls. The number of prescription drugs was higher among cases than among controls (mean difference $1.2,95 \%$ CI 1.16-1.26). While adjusting for potential confounders, we found that the risk of injurious falls increased in a nearly linear fashion for each additional drug (OR, 1.02; 95\% CI, 1.01-1.03). When using a cut-off value of $\geq 4$ drugs to define polypharmacy, the population attributable fraction for injurious falls was $5.2 \%$ (95\% CI 2.8-7.6). Conclusion: This study shows a monotonic dose-response relationship between the number of drugs and the risk of injurious falls. However, after comprehensive adjustment for known confounders (including fall-risk increasing drugs and chronic multimorbidity), this association is substantially weaker than previously reported. Moreover, even if the relationship between polypharmacy and injurious falls is really causal, the population attributable risk fraction is low.

Keywords: polypharmacy, falls, case-control, older people

\section{Introduction}

Sweden has one of the highest incidence rates of fall-related injuries and fatal falls among older adults in Europe. ${ }^{1}$ Falls often cause serious physical injuries (eg fractures), that in turn lead to functional impairment, disability, reduced social activities, lowered quality of life, and increased risk of mortality. ${ }^{2,3}$ In the United States, it is estimated that fatal and nonfatal falls among older adults result in medical costs reaching $\$ 50.0$ billion annually. ${ }^{4}$ Several factors have been identified as fall-risk increasing in older adults, including female sex, older age, chronic multimorbidity, muscle weakness, difficulties with gait and balance, poor vision, history of stroke, Parkinson's disease, dementia, epilepsy, orthostatic hypotension, depression, alcohol consumption, and falls. ${ }^{5,6}$ Besides, prior studies have shown that specific drug classes (eg antihypertensives, analgesics, sedatives, antidepressants, drugs with strong anticholinergic proprieties) can increase the risk of falls and fall-related injuries. ${ }^{7,8}$ Benzodiazepines and non-benzodiazepine hypnotics are of
Correspondence: Lucas Morin Aging Research Center, Karolinska Institutet, Tomtebodavägen 18A, Stockholm 17177, Sweden

$\mathrm{Tel}+46722887094$

Email lucas.morin@ki.se 
particular concern. ${ }^{9}$ The mechanisms underlying the effect of these so-called "fall-risk increasing drugs" (FRIDs) are mainly related to the secondary effects of each of these drug classes, eg imbalance, dizziness, reduced attention and vigilance, or mobility difficulties. ${ }^{7,10}$

Some studies have also suggested that beyond the effect of FRIDs, the mere number of prescribed medications may also increase the risk of falls in older people. $^{11,12}$ In a case-control study, Laflamme et al reported that the odds of injurious falls were 1.8 times greater among individuals prescribed $\geq 10$ drugs compared to those with only 1 drug. ${ }^{13}$ Cohort studies with follow-up periods ranging from 2 months to 3 years found similar results. ${ }^{14-18}$ In a large prospective cohort of 537387 older adults, polypharmacy ( $\geq 5$ drugs) at baseline was associated with an almost twofold increase in the incidence of falls over the first year of follow-up. ${ }^{19}$ This strong association between polypharmacy and the risk of falls could stem from the physiologically detrimental effects of multiple drug use on older adults' physical and cognitive functioning. ${ }^{20-22}$ However, this association may also be due to confounding-by-indication bias since polypharmacy is most often the consequence of chronic multimorbidity. ${ }^{23}$ In a systematic review and metaanalysis, Seppala et al found that only 19 out of 30 studies showed a positive association between polypharmacy and falls, with considerable heterogeneity in the observed estimates and with a majority of studies that did not appropriately adjust for the concomitant use of fall-risk increasing drugs and for chronic multimorbidity. ${ }^{24}$ Moreover, few studies included institutionalised older adults although polypharmacy is highly prevalent in the nursing home setting and these individuals may be particularly vulnerable to drug-related adverse events. ${ }^{25}$

Our study aimed to answer the following question: does the association between the number of prescribed drugs and the risk of injurious fall remain after adjusting for important confounders such as the use of fall-risk increasing drugs and chronic multimorbidity?

\section{Methods}

\section{Study design and data source}

We designed a matched nested case-control study based on routinely collected data with national coverage in Sweden. The study population was drawn from the entire population of older adults aged $\geq 70$ years and alive on 1 January 2013 . Data were linked at the individual level by Statistics Sweden and by the Swedish Board of Health and Welfare by using the personal identity number, ${ }^{26}$ and was subsequently anonymised.

\section{Selection of cases and controls}

Cases were defined as individuals who had a fall-related, non-elective hospital or emergency department admission between 1 January and 31 December 2013, considering the first admission for individuals who had repeated events during the study period. Hospital admissions and emergency department visits were identified through the Swedish National Patient Register, using International Classification of Diseases, 10th revision (ICD-10) codes W00 to W19. This register collects more than $99 \%$ of all inpatient admissions, and falls have been found to be correctly classified in over $98 \%$ of the cases. ${ }^{27}$ Controls with no fall-related injury during the study period were then randomly selected from the total Swedish population, and were matched 1:1 on sex, age, and index date (ie the date of admission for their matched case). The matching procedure is illustrated in Figure S1.

\section{Assessment of the main exposure}

The number of prescription drugs during the 7-day period preceding (but not including) the index date was considered as the main exposure. This guarantees that the exposure is chronologically positioned before the outcome, and thus that the direction of the association between the number of drugs and fall-related hospital admissions is unambiguous. In sensitivity analyses, the period of exposure was extended to respectively 15 and 30 days before the index date. Drug utilisation was estimated based on data from the Swedish Prescribed Drugs Register, which collects data about all prescription drugs dispensed through community pharmacies in Sweden since $2005 .^{28}$ The dispensing date, the dispensed dosage and the prescribed daily dose were used to calculate periods of drug exposure and to estimate the number of drugs used concomitantly during the 7 days before the index date (Figure S2). Drugs were counted as chemical substances $\left(5^{\text {th }}\right.$ level of the Anatomical Therapeutic Chemical [ATC] system). We categorised the number of drugs into 5 classes: $0-3$ drugs, 4-6 drugs, 7-9 drugs, 10-14 drugs, and $\geq 15$ drugs. Vaccines, diagnostic agents, nutrients and non-therapeutic products were not included.

\section{Fall-risk increasing drugs (frids)}

A total of 13 fall-risk increasing drugs were selected based on a review of the literature. ${ }^{7,29}$ They were supplemented with 10 drug classes with clinically relevant anticholinergic 
properties. The list of ATC codes and drugs is provided in Table S1. To ensure parallelism with the timeframe of the exposure, we counted the number of FRIDs during the 7-day period before the index date.

\section{Other covariates}

Potential confounders were identified a priori in the literature. ${ }^{5}$ Living arrangement was defined as either "community" or "nursing home", using data from the Swedish National Board of Health and Welfare's Social Services Register. This register contains information about care and services provided by municipalities to older adults and persons with functional impairment. To avoid reversed association, we assessed living arrangement during the last day of the month preceding the index date. The burden of chronic diseases was measured based on a recently proposed methodology, which encompasses a broad range of clinically relevant chronic conditions. ${ }^{30}$ We considered ICD-10 diagnoses reported in inpatient and specialised outpatient care facilities during a period of 5 years before the index date, starting from 31 days before the index date in order to avoid cross-contamination with the main exposure. The medical diagnoses were completed by a subset of specific medications dispensed during the same period, in order to increase the detection of chronic diseases often under-reported in the National Patient Register (eg dementia, diabetes, COPD). In one of the sensitivity analyses, we replaced this chronic multimorbidity assessment instrument by the Charlson Comorbidity Index score. ${ }^{31}$ History of fall-related hospitalisation (ICD-10 codes W00-W19) and history of alcohol-related hospitalisation (ICD-10 codes F10, K70, K860, K852, Y18, Y90, and Y91) during the 5-year period before the index date was assessed in the National Patient Register. Drug dispensing scheme was defined as "ordinary drug dispensing" or "multidose drug dispensing". The latter is a dose administration aid where medications are supplied to the patients in machine-packaged disposable plastic pouches. ${ }^{32}$ Previous studies have suggested an association between multidose drug dispensing and patterns of drug utilisation. ${ }^{33}$ Marital status was extracted from the National Patient Register (available for $97.6 \%$ of study participants). The level of education was assessed through the Swedish Register of Education, ${ }^{34}$ and was categorised as "primary", "secondary", and "tertiary" education based on the International Standard for Classification of Education (ISCED).

\section{Statistical analysis}

We assessed the association between the number of drugs and the risk of fall-related hospital admission using conditional logistic regression models. ${ }^{35,36}$ The first model was matched on sex and age, with no adjustment for additional confounders. In the second model, we repeated the analysis while controlling for the number of FRIDs during the 7 days before the index date. The third model was further adjusted on the living arrangement, the number of chronic diseases, previous fall-related hospital admissions, and the history of alcohol-related hospital admission. The number of drugs was primarily modelled as a continuous exposure by fitting restricted cubic splines with knots at 3, 5, 8, and 13 drugs to account for a potentially non-linear association. The number of drugs was also considered as a categorical variable, using the 5 classes mentioned earlier. We conducted four types of sensitivity analyses. First, we used unconditional logistic regressions adjusted for sex and age instead of a conditional models. Second, we adjusted for other potentially relevant confounders (ie education, marital status, multidose drug dispensing, and Charlson Comorbidity Index), based on previous studie. Third, the period of drug exposure was extended to respectively 15 and 30 days instead of the 7-day exposure window that was used in the main analysis. Finally, we restricted the analysis to fall-related hospital admissions with severe injuries (list of ICD-10 codes in Table S2). The main analysis was also stratified by sex, use of FRIDs, and living arrangement. Results are reported as odds ratio (OR) with 95\% confidence intervals (CI). Of note, since our study includes incident cases during the study period and since controls were sampled from a dynamic population and were matched on time, odds ratios can be interpreted as rate ratios. ${ }^{37}$ Finally, we estimated the population attributable fraction (PAF) to quantify the contribution of polypharmacy to the burden of injurious falls among older adults. This metric can be defined as the percentage reduction in incident fall-related hospital admissions that would be observed in the study population if polypharmacy was completely eliminated. ${ }^{38}$ It is calculated as $\mathrm{PAF}=P e \times[(\mathrm{OR}-1) \div \mathrm{OR}]$, where $P e$ is the prevalence of polypharmacy among cases. ${ }^{39,40}$ Because the interpretation of population attributable fraction is more straightforward with binary exposures, we dichotomised polypharmacy using 4 different cut-off 
values (4, 7, 10, and 15 drugs) and we stratified the analysis by age group. All analyses were performed on Stata version 14.1 (StataCorp, College Station, TX). Population attributable fraction was calculated with the PUNAFCC package. ${ }^{41}$ This study adheres to the RECORDPE guidelines (checklist provided as supplementary $\underline{\text { material). }}^{42}$

\section{Ethical approval}

The Regional Ethical Review Board in Stockholm approved the study (decision no. 2016/1001-31/4).

\section{Results}

\section{Characteristics of the study population}

A total of 49,609 older adults who had a fall-related hospital or emergency department admission in 2013 were included as cases and matched to an equal number of controls. Mean age at index date was 83.2 years (SD 7.2) among cases and 83.7 years (7.2) among controls. This difference in age can be attributed to the matching procedure, which assigned controls based on their attained age using rounded rather than exact values. As shown in Table 1, cases were more often living in nursing homes, had a higher number of chronic diseases, were more likely to have already been hospitalised for a fall and/or for alcohol-related conditions during the previous 5 years. Fall-risk increasing drugs were also more common among cases than among controls. Severe injuries were reported for $42852(86.4 \%)$ of all incident fall-related hospitalisations. The most common severe injuries involved the hip and thigh (28.7\%, including 15.5\% hip fractures), the elbow and forearm $(15.1 \%)$, the shoulder and upper arm $(12.6 \%)$, the head and neck (10.4\%), and the abdomen (9.1\%). A total of 2841 (5.7\%) cases died within 30 days after their admission.

\section{Association with polypharmacy}

The average number of prescription drugs during the week prior to the index date was higher among cases than among controls (6.4 vs 5.2, mean difference 1.2 , 95\% CI 1.16-1.26). The proportion of individuals exposed to $\geq 4$ drugs was also higher among cases than among controls (73.3\% vs $63.5 \%$ ). Matched analysis with no additional adjustment showed a monotonic dose-response association between the number of drugs and the risk of injurious fall. After controlling for the number of fall-risk increasing drugs during the exposure period, the estimates remained nearly identical. However, further adjustment for living arrangement, number of chronic diseases, history of fallrelated hospital admission, and history of alcohol-related hospital admission resulted in substantial effect size reductions (Figure 1). Assuming linearity, the adjusted risk of fall-related hospital admission was $2 \%$ higher for every increase of 1 drug (compared with $8 \%$ in the non-adjusted, matched analysis). There was no evidence of a multiplicative interaction with age, the level of education, the marital status, or the number of chronic diseases. However, the association between the number of drugs and the risk of fall-related hospital admission was somewhat weaker among women than among men (odds ratio for the interaction between sex and number of drugs: $0.97,95 \%$ CI 0.97-0.98). Stratified results by gender are presented in Table S3. As shown in Table 2, the adjusted odds ratios for fall-related hospital admission increased substantially with the number of drugs (OR 1.47, 95\% CI 1.32-1.64 for $\geq 15$ drugs compared to $0-3$ drugs).

\section{Population attributable fraction}

Figure 2 shows the population attributable fraction (PAF). The contribution of polypharmacy to the burden of fallrelated hospital admissions among older adults ranged from 5.2\% (95\% CI 2.8-7.6) when we considered a cutoff value of $\geq 4$ drugs to define polypharmacy to $0.6 \%$ (95\% CI 0.2-0.9) when considering a cut-off value of $\geq 15$ drugs. PAF was lowest among individuals aged 90 years and older, although the prevalence of polypharmacy was similar to that of younger age groups.

\section{Sensitivity analyses}

Restricting the main analysis to only fall-related hospital admissions resulting in severe injuries did not change the results (Table S4), nor did using unconditional logistic regressions adjusted for sex and age instead of conditional models (Table S5). After adjustment on the level of education and the marital status of study participants, the association between the number of drugs and the risk of injurious fall remained very similar to that of our primary analysis. However, controlling for multidose dispensing moderated this association. Conversely, controlling for the burden of chronic diseases through the Charlson Comorbidity Index led to a substantially larger effect size of polypharmacy than in the main analysis (Table S6). Extension of the drug exposure period before the index date had very little effect on the results (Table S7). A subgroup analysis revealed that whereas the association between the number of drugs and the risk of 
Table I Characteristics of the study population

\begin{tabular}{|c|c|c|}
\hline & Cases $(\mathrm{N}=49,609)$ & Controls $(N=49,609)$ \\
\hline \multicolumn{3}{|l|}{ Sex, No. (\%) } \\
\hline Men & $15,285(30.8)$ & $15,285(30.8)$ \\
\hline Women & $34,324(69.2)$ & $34,324(69.2)$ \\
\hline \multicolumn{3}{|l|}{ Age, years } \\
\hline Mean (SD) & $83.2(7.2)$ & $83.7(7.2)$ \\
\hline No. (\%) & & \\
\hline 70-79 years & $16,237(32.7)$ & I5,368 (31.0) \\
\hline $80-89$ years & $22,728(45.8)$ & $22,532(45.4)$ \\
\hline$\geq 90$ years & $10,644(21.5)$ & II,709 (23.6) \\
\hline \multicolumn{3}{|c|}{ Living arrangement, No. (\%) } \\
\hline Community & $42,818(86.3)$ & $44,054(88.8)$ \\
\hline Nursing home & $6,79 \mid(13.7)$ & $5,555(11.2)$ \\
\hline \multicolumn{3}{|l|}{ Marital status ${ }^{\mathrm{a}}$, No. (\%) } \\
\hline Single & $3,463(7.0)$ & $2,924(6.2)$ \\
\hline Married & $|5,79|(3 \mid .9)$ & $17,276(36.4)$ \\
\hline Divorced & $7,377(14.9)$ & $5,843(12.3)$ \\
\hline Widowed & $22,848(46.2)$ & $21,36 I(45.1)$ \\
\hline \multicolumn{3}{|c|}{ Level of education ${ }^{\mathrm{b}}$, No. (\%) } \\
\hline Primary education & $22,057(45.4)$ & $21,676(45.1)$ \\
\hline Secondary education & $19,443(40.0)$ & $19,013(39.6)$ \\
\hline Tertiary education & $7,103(14.6)$ & $7,375(15.3)$ \\
\hline \multicolumn{3}{|c|}{ Fall-risk increasing drugs } \\
\hline Mean (SD) & $2.5(2.0)$ & $2.0(1.7)$ \\
\hline No. (\%) & & \\
\hline 0 & $8,488(I 7.1)$ & II,565 (23.3) \\
\hline I & $8,799(I 7.7)$ & $9,935(20.0)$ \\
\hline$\geq 2$ & $32,322(65.2)$ & $28,109(56.7)$ \\
\hline \multicolumn{3}{|c|}{ Number of chronic diseases } \\
\hline Mean (SD) & $4.9(3.4)$ & $3.8(3.0)$ \\
\hline \multicolumn{3}{|l|}{ No. (\%) } \\
\hline 0 & $3,167(6.4)$ & $5,344(10.8)$ \\
\hline I & $4,623(9.3)$ & $6,822(\mid 3.8)$ \\
\hline 2 & $5,609(I 1.3)$ & $7,252(\mid 4.6)$ \\
\hline$\geq 3$ & $36,210(73.0)$ & $30,191(60.9)$ \\
\hline \multicolumn{3}{|c|}{ History of fall-related hospitalisation, No. (\%) } \\
\hline Yes & $13,134(26.5)$ & $7,649(15.4)$ \\
\hline No & $36,475(73.5)$ & $41,960(84.6)$ \\
\hline \multicolumn{3}{|c|}{$\begin{array}{l}\text { History of alcohol-related } \\
\text { hospitalisation, No. (\%) }\end{array}$} \\
\hline Yes & $\mathrm{I}, 003(2.0)$ & $259(0.5)$ \\
\hline No & $48,606(98.0)$ & $49,350(99.5)$ \\
\hline \multicolumn{3}{|c|}{ Drug dispensing scheme } \\
\hline Ordinary prescriptions & $36,270(73.1)$ & $40,818(82.3)$ \\
\hline Multidose dispensing & 13,339 (26.9) & $8,79 \mid(17.7)$ \\
\hline
\end{tabular}

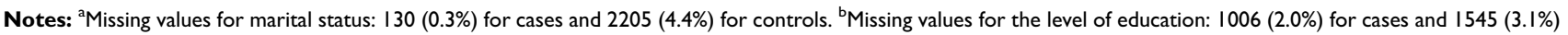
for controls 


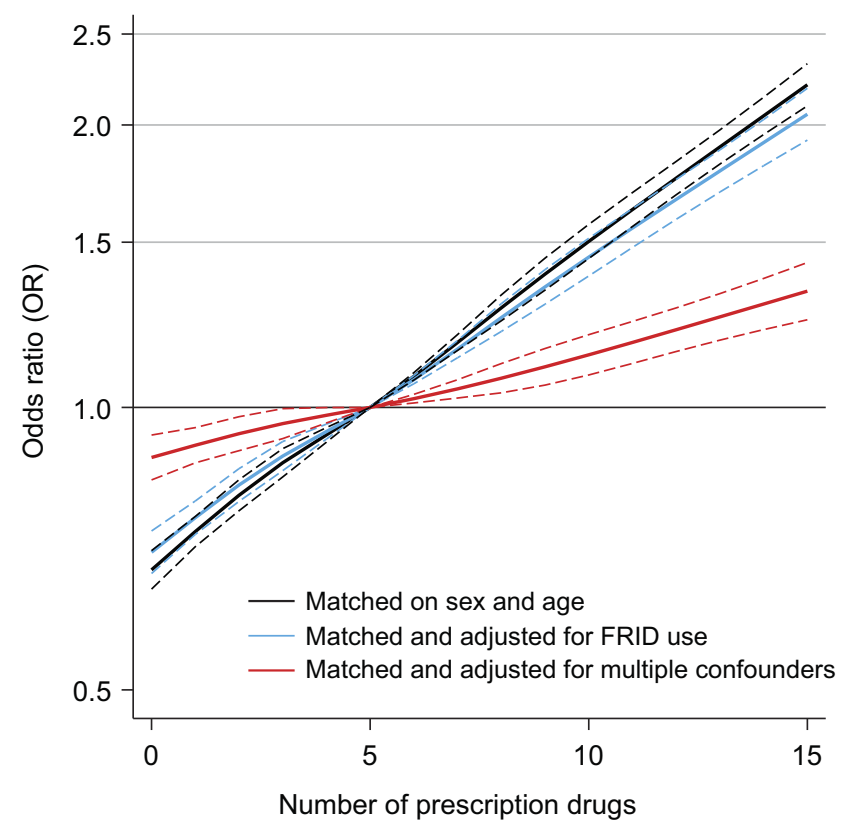

Figure I Dose-response relationship between the number of prescription drugs and the risk of injurious fall.

Notes: Odds ratios for fall-related hospital admissions modelled by restricted cubic spline models. The number of prescription drugs was transformed using restricted cubic regression splines with knots at 3, 5, 8 and 13 drugs. Conditional logistic regression models were then fitted to estimate odds ratio (solid curves) with pointwise $95 \%$ confidence intervals (dashed curves). The median number of drugs (5) was chosen as reference point. The fully adjusted model was matched on sex, age and index date, and further adjusted for the number of fall-risk increasing drugs (FRIDs), living arrangement, number of chronic diseases, history of fall-related hospital admission, and history of alcohol-related hospital admission. Assuming linearity, an odds ratio of $1.02(95 \% \mathrm{Cl}$ I.0I-I.03) was observed for every increase of I drug during the 7-day period before (but not including) the index date.

Abbreviation: FRID, fall-risk increasing drug.

injurious fall was similar among FRID users and non-users (3\% increased odds for each additional drug), this association was no longer statistically significant above a threshold of 7 drugs (Table S8). Finally, we found that the association between the number of drugs and the risk of injurious fall could be observed among community dwellers but not among nursing home residents (Table S9).

\section{Discussion}

This nationwide nested case-control study shows that there is a monotonic dose-response relationship between the number of drugs and the risk of injurious falls. However, this association is substantially weaker than previously reported. After controlling for relevant confounders (including fall-risk increasing drugs and chronic multimorbidity), the contribution of polypharmacy to the burden of injurious falls among older adults appears to be low.

Identifying whether or not the association between polypharmacy and falls is causal is essential to determine if future interventions targeting polypharmacy can reduce the burden of fall-related injuries among older adults. Causality is more probable if the association between the exposure and the outcome follows a gradient such as the more intense the exposure, the greater the risk of the outcome. ${ }^{43}$ This assumption is supported by the clear dose-response relationship that we observed between the number of drugs and the risk of fall-related hospital admissions, even after controlling for a broad range of known confounders. On average, the risk of injurious falls increases by $2 \%$ for each additional drug, in a remarkably linear fashion. Although of substantially lower magnitude, this observed association between polypharmacy and injurious falls is in keeping with the results from a recent meta-analysis ${ }^{24}$ and from a longitudinal cohort study of older adults in England. ${ }^{12}$ In other words, despite substantial methodological heterogeneity, studies conducted in different countries and different patient groups reach similar conclusions. This consistency across studies adds weight to the hypothesis of a causal effect of polypharmacy. Our finding that the association between polypharmacy and injurious falls is weaker than previously reported may stem from differences in the outcome definition. While many population-based studies included self-reported (injurious as well as non-injurious) falls, the present study was based solely on routinely collected healthcare data and could therefore only identify falls that led to a hospital admission or an emergency department visit.

Adequate adjustment for common causes of the exposure and the outcomes is critical to meet the assumption of exchangeability in observational studies. We believe that important confounders may have been overlooked in the past. For instance, the burden of anticholinergic drugs and the history of fall-related injuries have often not been accounted for. ${ }^{11,24}$ Our finding that there was no association between the number of prescription drugs and the risk of fall-related injuries among nursing home residents also suggests that the determinants of injurious falls among institutionalised older persons are different than those identified in the community. Moreover, since chronic multimorbidity increases both the probability to be prescribed multiple medications and the probability of having an injurious fall, confounding-by-indication is a serious concern. In sensitivity analyses, controlling for Charlson Comorbidity Index instead of the more extensive assessment tool used in our main model resulted in substantially larger effect sizes for the association between 


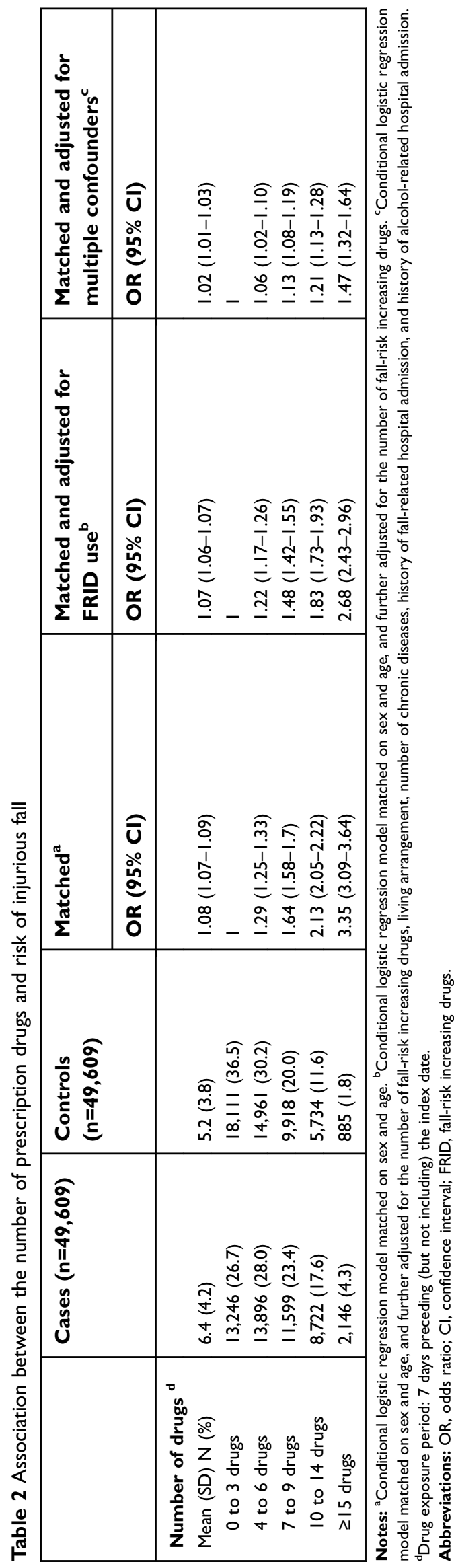

polypharmacy and odds of injurious falls. This suggests that studies relying on insufficiently comprehensive instruments for measuring the burden of chronic diseases in old age may overestimate the effect of polypharmacy because of residual "confounding-by-multimorbidity".

Finally, the issue of temporality must be appropriately addressed to establish a causal relationship between polypharmacy and adverse health outcomes. Beyond the traditional criteria of chronology (the exposure must precede the outcome), we argue that longitudinal studies concerned with demonstrating the existence of a causal effect of polypharmacy on adverse health outcomes should also make sure that these outcomes occur during a period when the subjects are indeed exposed to polypharmacy or very shortly afterwards (criteria of temporal proximity). Contrary to chronic conditions, polypharmacy is not necessarily an absorbing state. Many older adults are exposed to transient episodes of polypharmacy caused by either an acute illness (eg infectious diseases) or temporary symptoms (eg analgesics after surgery). ${ }^{44}$ Among these individuals, it seems biologically implausible that the exposure to polypharmacy during a given week could have an impact on their risk of falling 12,18 or 24 months later. Yet, most studies published to date have relied on cohort designs comparing the outcomes of older adults during follow-up according to their number of prescribed drugs at baseline. Little or no consideration has been given the time-varying nature of drug therapy, and thus of the exposure to polypharmacy. ${ }^{11,45-47}$ In the present study, the prerequisite of temporal proximity mentioned above was the main reason why we chose a nested case-control design with well-defined windows of exposure to polypharmacy.

The current discussion about the potential impact of polypharmacy on fall-related injuries among older adults relies on two cumulative hypotheses: first, that it possible to reduce the prevalence of polypharmacy in the older population; and second, that reducing polypharmacy would substantially decrease the incidence of fall-related injuries. Recent empirical studies have challenged the first assumption by showing that polypharmacy is often justified by the complex clinical profile of older adults, and that interventions to reduce polypharmacy are largely unsuccessful. ${ }^{4-52}$ Our findings challenge the second hypothesis. Indeed, even if polypharmacy had a truly causal effect on injurious falls and even if successful interventions could achieve a complete reduction of polypharmacy in the older population, the gains in terms of fall incidence would be low (PAF ranging from 0.1 to 5\%). Therefore, the present study has important implications for healthcare professionals, stakeholders and policy-makers who strive to 


\begin{tabular}{|c|c|c|c|c|}
\hline Subgroup & $\begin{array}{l}\text { Prevalence } \\
\text { among cases }\end{array}$ & $\begin{array}{l}\text { Odds ratio } \\
\text { for injurious falls }(95 \% \mathrm{Cl})^{\mathrm{a}}\end{array}$ & & $\operatorname{PAF}(95 \% \mathrm{Cl})^{\mathrm{b}}$ \\
\hline \multicolumn{5}{|c|}{ All cases and controls } \\
\hline$\geq 4$ drugs & $73.3 \%$ & 1.08 (1.04 to 1.12$)$ & a & $5.2 \%$ (2.8 to 7.6$)$ \\
\hline$\geq 7$ drugs & $45.3 \%$ & 1.09 (1.05 to 1.13$)$ & a & $3.8 \%$ (2.3 to 5.3$)$ \\
\hline$\geq 10$ drugs & $21.9 \%$ & 1.07 (1.03 to 1.12$)$ & |ө| & $1.5 \%(0.6$ to 2.4$)$ \\
\hline$\geq 15$ drugs & $4.3 \%$ & 1.15 (1.06 to 1.26$)$ & $1 \bullet-1$ & $0.6 \%(0.2$ to 0.9$)$ \\
\hline \multicolumn{5}{|l|}{ 70-79 years } \\
\hline$\geq 4$ drugs & $64.1 \%$ & 1.04 (0.98 to 1.10$)$ & 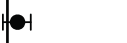 & $2.3 \%(-1.6$ to 6.0$)$ \\
\hline$\geq 7$ drugs & $37.3 \%$ & 1.11 (1.04 to 1.19$)$ & |-1 & $3.7 \%$ (1.4 to 6.0$)$ \\
\hline$\geq 10$ drugs & $18.4 \%$ & 1.15 (1.06 to 1.26$)$ & 1-1 & $2.5 \%$ (1.0 to 3.9$)$ \\
\hline$\geq 15$ drugs & $4.4 \%$ & 1.31 (1.10 to 1.56$)$ & $\longmapsto$ & $1.1 \%(0.4$ to 1.7$)$ \\
\hline \multicolumn{5}{|l|}{ 80-89 years } \\
\hline$\geq 4$ drugs & $77.7 \%$ & 1.08 (1.02 to 1.14$)$ & |өr & $5.7 \%$ (1.7 to 9.4$)$ \\
\hline$\geq 7$ drugs & $49.3 \%$ & $1.13(1.07$ to 1.19$)$ & 101 & $5.5 \%$ (3.2 to 7.8 ) \\
\hline$\geq 10$ drugs & $24.0 \%$ & $1.08(1.01$ to 1.15$)$ & $\bullet-1$ & $1.7 \%(0.3$ to 3.1$)$ \\
\hline$\geq 15$ drugs & $4.5 \%$ & $1.10(0.98$ to 1.25$)$ & $\mapsto \bullet-1$ & $0.4 \%(-0.1$ to 0.9$)$ \\
\hline \multicolumn{5}{|c|}{90 years and older } \\
\hline$\geq 4$ drugs & $79.2 \%$ & 1.06 (0.98 to 1.15$)$ & to-1 & $4.6 \%(-1.5$ to 10.4$)$ \\
\hline$\geq 7$ drugs & $49.9 \%$ & 1.00 (0.93 to 1.08$)$ & & $0.1 \%(-3.6$ to 3.7$)$ \\
\hline$\geq 10$ drugs & $23.2 \%$ & $1.02(0.94$ to 1.11$)$ & H & $0.4 \%(-1.5$ to 2.4$)$ \\
\hline \multirow[t]{2}{*}{$\geq 15$ drugs } & $3.6 \%$ & 1.09 (0.91 to 1.31$)$ & -1 & $0.3 \%(-0.3$ to 0.9$)$ \\
\hline & & 0.5 & 1.0 & \\
\hline
\end{tabular}

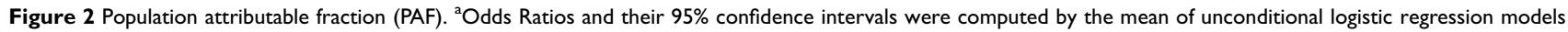
adjusted for sex, age, number of fall-risk increasing drugs (FRIDs), living arrangement, number of chronic diseases, history of fall-related hospital admission, and history of alcohol-related hospital admission. ${ }^{\mathrm{b}}$ The population attributable fraction (PAF) can be calculated as $\mathrm{PAF}=\mathrm{Pe} \times[(\mathrm{OR}-\mathrm{I}) \div \mathrm{OR}]$, where $\mathrm{Pe}$ is the prevalence rate of the exposure among cases.

find effective interventions to substantially reduce the burden of morbidity generated by falls in old age. This does not mean that deprescribing strategies are not warranted to minimise the treatment burden and to avoid the harms caused by drug-drug interactions and adverse drug reactions, but the effectiveness (and cost-effectiveness) of these strategies to improve fall rates is uncertain, ${ }^{53,54}$ especially compared to interventions targeting high-risk drug classes ${ }^{55,56}$ or promoting physical activity. ${ }^{57}$

Strengths of this study include the use of high-quality data with national coverage in Sweden, thus minimising selection bias and increasing the generalisability of our findings. The objective ascertainment of fall-related hospital admissions also reduces the risk of recall bias and outcome misclassification that commonly threatens the validity of case-control studies. In addition, the estimation of drug exposure during the week immediately before the outcome increases the biological plausibility of the observed association between polypharmacy and fall-related hospital admission. Nevertheless, this study should be examined carefully in light of its methodological limitations. Although our approach to constructing drug exposure periods based on the prescribed daily dose is more accurate than methodologies relying solely on defined daily doses, ${ }^{58}$ the estimated burden of drug exposure during the week before the index date relies on the (unverifiable) assumption that patients ingested the drugs as prescribed. In addition, the Swedish Prescribed Drugs Register does not collect data about overthe-counter drugs, drugs administered within hospitals, and drugs dispensed through nursing home storerooms. These limitations could lead to some degree of misclassification of the actual exposure to polypharmacy. ${ }^{59}$ Moreover, we did not take the dosage of each drug into account, thereby ignoring the fact that stronger doses of fall-risk increasing drugs or anticholinergics may increase the risk of falls. There also exists a risk of protopathic bias: the drugs composing polypharmacy could very well have been initiated in response to underlying, subclinical symptoms of the actual cause for the falls (eg opioids for pain). ${ }^{60}$ Finally, the observed association between polypharmacy and fallrelated hospitalisation is most likely affected by residual, unmeasured confounding related to deficits in physical functions and cognitive performance that studies relying on routinely collected data can seldom account for. ${ }^{61}$ 


\section{Conclusion}

Although the present study shows a monotonic dose-response relationship between the number of prescription drugs and the subsequent risk of injurious fall, this association is substantially weaker than previously reported. This highlights the need for adequate strategies to address the challenge of "confounding by multimorbidity" in observational studies investigating the potential harms of polypharmacy. Moreover, even if polypharmacy had a truly causal effect, the population attributable risk does not surpass 5\%. Our findings thus suggest that despite justifiable concerns about the hazards of polypharmacy at the patient level, interventions aiming to reduce polypharmacy per se are unlikely to substantially reduce the burden of injurious fall-related in the older population.

\section{Availability of data and materials}

Patient-level data from the Swedish Prescribed Drugs Register and other healthcare registers cannot be made publicly available. Interested researchers can access the aggregated data from the Swedish Prescribed Drugs Register (www.socialstyrelsen.se/statistik/statistikdatabas/ lakemedel). Additional data can be obtained upon reasonable request to the authors.

\section{Ethical statement}

The study was approved by the Ethical Review Board in Stockholm, Sweden and was carried out in accordance with the principles of the Declaration of Helsinki.

\section{Acknowledgments}

The authors are grateful to Johan Fastbom for providing the software used to compute the exposure to polypharmacy. This work was supported by grants from the Swedish Research Council for Health, Working Life and Welfare and from the Swedish Research Council. The funders had no role in study design, data collection and analysis, decision to publish, or preparation of the manuscript.

\section{Author contributions}

LM conceived and designed the study, performed the statistical analysis, interpreted the data, drafted, and critically revised the manuscript. ACL, AKW, DR and JWW interpreted the data and critically revised the manuscript. $\mathrm{KJ}$ obtained funding, provided supervision, interpreted the data and, critically revised the manuscript. LM is the guarantors of the study and data integrity.
All authors gave approval for the final version of the manuscript and agree to be accountable for all aspects of the work.

\section{Disclosure}

The authors declare that they have no conflicts of interest regarding this article.

\section{References}

1. Lucas R, Martins A, Severo M, et al. Quantitative modelling of hip fracture trends in 14 European countries: testing variations of a shared reversal over time. Sci Rep. 2017;7(1):3754. doi:10.1038/s41598-017-03847-x

2. Verma SK, Willetts JL, Corns HL, et al. Falls and fall-related injuries among community-dwelling adults in the United States. PLoS One. 2016;11(3):1-14. doi:10.1371/journal.pone.0150939

3. Gill TM, Murphy TE, Gahbauer EA, Allore HG. Association of injurious falls with disability outcomes and nursing home admissions in community-living older persons. Am J Epidemiol. 2013;178 (3):418-425. doi:10.1093/aje/kws554

4. Florence CS, Bergen G, Atherly A, Burns E, Stevens J, Drake C. Medical costs of fatal and nonfatal falls in older adults. J Am Geriatr Soc. 2018;66(4):693-698. doi:10.1111/jgs.15304

5. Deandrea S, Lucenteforte E, Bravi F, Foschi R, La Vecchia C, Negri E. Risk factors for falls in community-dwelling older people: a systematic review and meta-analysis. Epidemiology. 2010;21 (5):658-668. doi:10.1097/EDE.0b013e3181e89905

6. Ek S, Rizzuto D, Fratiglioni L, Johnell K, Xu W, Welmer AK. Risk profiles for injurious falls in people over 60: a population-based cohort study. J Gerontol A Biol Sci Med Sci. 2018;73(2):233-239. doi:10.1093/gerona/glx115

7. Woolcott JC, Richardson KJ, Wiens MO, et al. Meta-analysis of the impact of 9 medication classes on falls in elderly persons. Arch Intern Med. 2009;169(21):1952-1960. doi:10.1001/archinternmed.2009.357

8. Tinetti ME, Han L, Lee DSH, et al. Antihypertensive medications and serious fall injuries in a nationally representative sample of older adults. JAMA Intern Med. 2014;174(4):588-595. doi:10.1001/ jamainternmed.2013.14764

9. Johnell K, Jonasdottir Bergman G, Fastbom J, Danielsson B, Borg N, Salmi P. Psychotropic drugs and the risk of fall injuries, hospitalisations and mortality among older adults. Int J Geriatr Psychiatry. 2017;32 (4):414 420. doi:10.1002/gps.4483

10. Seppala LJ, Wermelink AMAT, de Vries M, et al. Fall-risk-increasing drugs: a systematic review and meta-analysis: II. psychotropics. J Am Med Dir Assoc. 2018;19(4):371.e11-371.e17. doi:10.1016/j. jamda.2017.12.098

11. Fried TR, O’Leary J, Towle V, Goldstein MK, Trentalange M, Martin DK. Health outcomes associated with polypharmacy in community-dwelling older adults: a systematic review. $J \mathrm{Am}$ Geriatr Soc. 2014;62(12):2261-2272. doi:10.1111/jgs.13153

12. Dhalwani NN, Fahami R, Sathanapally H, Seidu S, Davies MJ, Khunti K. Association between polypharmacy and falls in older adults: a longitudinal study from England. BMJ Open. 2017;7 (10):1-8. doi:10.1136/bmjopen-2017-016358

13. Laflamme L, Monárrez-Espino J, Johnell K, Elling B, Möller J, Icks A. Type, number or both? A population-based matched case-control study on the risk of fall injuries among older people and number of medications beyond fall-inducing drugs. PLoS One. 2015;10(3):e0123390. doi:10.1371/journal.pone.0123390

14. Kojima T, Akishita M, Nakamura T, et al. Polypharmacy as a risk for fall occurrence in geriatric outpatients. Geriatr Gerontol Int. 2012;12 (3):425-430. doi:10.1111/j.1447-0594.2011.00783.x 
15. Bergland A, Jarnlo GB, Laake K. Predictors of falls in the elderly by location. Aging Clin Exp Res. 2003;15(1):43-50.

16. Clough-Gorr KM, Erpen T, Gillmann G, et al. Preclinical disability as a risk factor for falls in community-dwelling older adults. J Gerontol A Biol Sci Med Sci. 2008;63(3):314-320. doi:10.1093/gerona/63.3.314

17. Pluijm SMF, Smit JH, Tromp EAM, et al. A risk profile for identifying community-dwelling elderly with a high risk of recurrent falling: results of a 3-year prospective study. Osteoporos Int. 2006;17 (3):417-425. doi:10.1007/s00198-005-0002-0

18. Bennett A, Gnjidic D, Gillett M, et al. Prevalence and impact of fall-riskincreasing drugs, polypharmacy, and drug-drug interactions in robust versus frail hospitalised falls patients: a prospective cohort study. Drugs Aging. 2014;31(3):225-232. doi:10.1007/s40266-013-0151-3

19. Nishtala PS, Narayan SW, Wang T, Hilmer SN. Associations of drug burden index with falls, general practitioner visits, and mortality in older people. Pharmacoepidemiol Drug Saf. 2014;23(7):753-758. doi: $10.1002 /$ pds.3624

20. Langeard A, Pothier K, Morello R, et al. Polypharmacy cut-off for gait and cognitive impairments. Front Pharmacol. 2016;7:296. doi:10.3389/fphar.2016.00323

21. George C, Verghese J. Polypharmacy and gait performance in community-dwelling older adults. $J$ Am Geriatr Soc. 2017;65 (9):2082-2087. doi:10.1111/jgs.14957

22. Rawle MJ, Cooper R, Kuh D, Richards M. Associations between polypharmacy and cognitive and physical capability: a british birth cohort study. J Am Geriatr Soc. 2018. Epub. doi:10.1111/ jgs. 15317

23. Morin L, Johnell K, Laroche M-L, Fastbom J, Wastesson JW. The epidemiology of polypharmacy in older adults: register-based prospective cohort study. Clin Epidemiol. 2018;10:289-298. doi:10.2147/CLEP.S153458

24. Seppala LJ, van de Glind EMM, Daams JG, et al. Fall-Riskincreasing drugs: a systematic review and meta-analysis: III. Others. $J$ Am Med Dir Assoc. 2018;19(4):372.e1-372.e8. doi:10.1016/j.jamda.2017.12.099

25. Jokanovic N, Tan ECK, Dooley MJ, Kirkpatrick CM, Bell JS. Prevalence and factors associated with polypharmacy in long-term care facilities: a systematic review. J Am Med Dir Assoc. 2015;16 (6):535.e1-535.e12. doi:10.1016/j.jamda.2015.03.003

26. Ludvigsson JF, Otterblad-Olausson P, Pettersson BU, Ekbom A. The Swedish personal identity number: possibilities and pitfalls in healthcare and medical research. Eur J Epidemiol. 2009;24(11):659-667. doi:10.1007/s10654-009-9350-y

27. Bergström MF, Byberg L, Melhus H, Michaelsson K, Gedeborg R. Extent and consequences of misclassified injury diagnoses in a national hospital discharge registry. Inj Prev. 2011;17(2):108-113. doi:10.1136/ip.2010.028951

28. Wettermark B, Hammar N, Fored CM, et al. The new Swedish prescribed drug register-opportunities for pharmacoepidemiological research and experience from the first six months. Pharmacoepidemiol Drug Saf. 2007;16(7):726-735. doi:10.1002/ pds. 1294

29. Ruxton K, Woodman RJ, Mangoni AA. Drugs with anticholinergic effects and cognitive impairment, falls and all-cause mortality in older adults: a systematic review and meta-analysis. $\mathrm{Br} \mathrm{J}$ Clin Pharmacol. 2015;80(2):209-220. doi:10.1111/bcp.12617

30. Calderón-Larrañaga A, Vetrano DL, Onder G, et al. Assessing and measuring chronic multimorbidity in the older population: a proposal for its operationalization. J Gerontol A Biol Sci Med Sci. 2017;72 (10):1417-1423.

31. Charlson ME, Pompei P, Ales KL, MacKenzie R. A new method of classifying prognostic in longitudinal studies: development and validation. J Chronic Dis. 1987;40:373-383.

32. Bell JS, Johnell K, Wimmer BC, Wiese MD. Multidose drug dispensing and optimising drug use in older people. Age Ageing. 2013;42 (5):556-558. doi:10.1093/ageing/aft080
33. Johnell K, Fastbom J. Multi-dose drug dispensing and inappropriate drug use: a nationwide register-based study of over 700000 elderly. Scand J Prim Health Care. 2008;26(2):86-91. doi:10.1080/ 02813430802022196

34. Statistics Sweden. Education of the Population. Örebro: Statistics Sweden (SCB); 2014.

35. Hosmer DW, Lemeshowr S, Sturdivantr RX. Logistic Regression for Matched Case-Control Studies. In: Hosmer DW, Lemeshowr S, Sturdivantr RX. Applied Logistic Regression. Hoboken, New Jersey: Wiley; 2013:243-268.

36. Pearce N. Analysis of matched case-control studies. BMJ. 2016;352: i969. doi:10.1136/bmj.i1717

37. Knol MJ, Vandenbroucke JP, Scott P, Egger M. What do case-control studies estimate? Survey of methods and assumptions in published case-control research. Am J Epidemiol. 2008;168(9):1073-1081. doi:10.1093/aje/kwn217

38. Mansournia MA, Altman DG. Population attributable fraction. BMJ. 2018;k757. doi:10.1136/bmj.k757

39. Coughlin SS, Benichou J, Weed DL. Attributable risk estimation in case-control studies. Epidemiol Rev. 1994;16(1):51-64. doi:10.1093/ oxfordjournals.epirev.a036144

40. Cole P, MacMahon B. Attributable risk percent in case-control studies. Br J Prev Soc Med. 1971;25(4):242-244. doi:10.1136/ jech.25.4.242

41. Newton HJ, Baum CF, Beck N, et al. Attributable and unattributable risks and fractions and other scenario comparisons. Stata J. 2013;13 (4):672-698. doi:10.1177/1536867X1301300402

42. Langan SM, Schmidt SA, Wing K, et al. The reporting of studies conducted using observational routinely collected health data statement for pharmacoepidemiology (RECORD-PE). BMJ;2018:k3532. doi:10.1136/bmj.k3532

43. Bradford-Hill A. The Enviroment and disease: association or causation? Proc R Soc Med. 1965;58:295-300.

44. Wastesson JW, Morin L, Laroche M-L, Johnell K. How chronic is polypharmacy in old age? A longitudinal nationwide cohort study. J Am Geriatr Soc. 2019;67(3):455-462. doi:10.1111/jgs.15717

45. Schlesinger A, Weiss A, Nenaydenko O, Adunsky A, Beloosesky Y. Does polypharmacy in nursing homes affect long-term mortality? J Am Geriatr Soc. 2016;64(7):1432-1438. doi:10.1111/jgs.14213

46. Veronese N, Stubbs B, Noale M, et al. Polypharmacy is associated with higher frailty risk in older people: an 8-year longitudinal cohort study. J Am Med Dir Assoc. 2017;18(7):624-628. doi:10.1016/j. jamda.2017.02.009

47. Gnjidic D, Hilmer SN, Blyth FM, et al. Polypharmacy cutoff and outcomes: five or more medicines were used to identify community-dwelling older men at risk of different adverse outcomes. J Clin Epidemiol. 2012;65 (9):989-995. doi:10.1016/j.jclinepi.2012.02.018

48. Wise J. Polypharmacy: a necessary evil. BMJ. 2013;347:f7033f7033. doi:10.1136/bmj.f7033

49. Duerden M, Avery T, Payne R. Polypharmacy and medicines optimisation. Making it safe and sound. London: The King's Fund; 2013:168. Available from https://www.kingsfund.org.uk/publications/poly pharmacy-and-medicines-optimisation

50. Wallace E, Salisbury C, Guthrie B, Lewis C, Fahey T, Smith SM. Managing patients with multimorbidity in primary care. $\mathrm{Br} \mathrm{Med} J$. 2015;350(jan20 2):h176-h176. doi:10.1136/bmj.h176

51. Rankin A, Cadogan CA, Patterson SM, et al. Interventions to improve the appropriate use of polypharmacy for older people. Cochrane Database Syst Rev. 2018;9:CD008165. doi:10.1002/ 14651858.CD008165.pub4

52. Salisbury C, Man M, Bower P, et al. Management of multimorbidity using a patient-centred care model: a pragmatic cluster-randomised trial of the 3D approach. Lancet. 2018;6736(18):1-10.

53. Gillespie LD, Robertson MC, Gillespie WJ, et al. Interventions for preventing falls in older people living in the community. Cochrane Database Syst Rev. 2009;2(CD007146):CD007146. 
54. Dills H, Shah K, Messinger-Rapport B, Bradford K, Syed Q. Deprescribing medications for chronic diseases management in primary care settings: a systematic review of randomized controlled trials. J Am Med Dir Assoc. 2018. doi:10.1016/j.jamda.2018.06.021

55. Donnelly K, Bracchi R, Hewitt J, Routledge PA, Carter B Benzodiazepines, Z-drugs and the risk of hip fracture: a systematic review and meta-analysis. PLoS One. 2017;12(4). doi:10.1371/journal.pone. 0174730

56. Treves N, Perlman A, Geron LK, Asaly A, Matok I. Z-drugs and risk for falls and fractures in older adults-a systematic review and meta-analysis. Age Ageing. 2018;47(2):201-208. doi:10.1093/ageing/afx 167

57. de Souto-Barreto P, Rolland Y, Vellas B, Maltais M. Association of long-term exercise training with risk of falls, fractures, hospitalizations, and mortality in older adults. A systematic review and metaanalysis. JAMA Intern Med. 2019;179(3):394-405. doi:10.1001/ jamainternmed.2018.540
58. Sinnott S-J, Polinski JM, Byrne S, Gagne JJ. Measuring drug exposure: concordance between defined daily dose and days' supply depended on drug class. J Clin Epidemiol. 2016;69:107-113. doi:10.1016/j.jclinepi.2015.05.026

59. Gamble J-M, McAlister FA, Johnson JA, Eurich DT. Quantifying the impact of drug exposure misclassification due to restrictive drug coverage in administrative databases: a simulation cohort study. Value Heal. 2012;15(1):191-197. doi:10.1016/j. jval.2011.08.005

60. Horwitz RI, Feinstein AR. The problem of "protopathic bias" in casecontrol studies. Am J Med. 1980;68(2):255-258.

61. Welmer AK, Rizzuto D, Laukka EJ, Johnell K, Fratiglioni L. Cognitive and physical function in relation to the risk of injurious falls in older adults: a population-based study. J Gerontol A Biol Sci Med Sci. 2017;72(5):669-675.
Clinical Epidemiology

\section{Publish your work in this journal}

Clinical Epidemiology is an international, peer-reviewed, open access, online journal focusing on disease and drug epidemiology, identification of risk factors and screening procedures to develop optimal preventative initiatives and programs. Specific topics include: diagnosis, prognosis, treatment, screening, prevention, risk factor modification,

Submit your manuscript here: https://www.dovepress.com/clinical-epidemiology-journal
Dovepress

systematic reviews, risk \& safety of medical interventions, epidemiology \& biostatistical methods, and evaluation of guidelines, translational medicine, health policies \& economic evaluations. The manuscript management system is completely online and includes a very quick and fair peer-review system, which is all easy to use. 